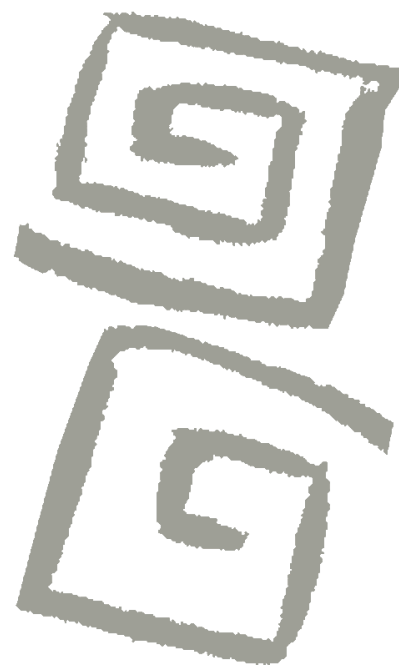

\title{
Distribución de caries dental y asociación con variables de protección social en niños de 12 años del partido de Avellaneda, provincia de Buenos Aires
}

\author{
Distribution of dental caries and its association with \\ variables of social protection in children 12 years of age \\ in the county of Avellaneda, Province of Buenos Aires
}

Adriana Fort ${ }^{1}$, Aida Julia Fuks², Alberto Vicente Napoli ${ }^{3}$, Silvia Palomba ${ }^{4}$, Ximena Pazos ${ }^{5}$, Pablo Salgado ${ }^{6}$, Graciela Klemonskis ${ }^{7}$, Aldo Squassi ${ }^{8}$

'Odontóloga, Especialista en Odontopediatría. Ayudante de primera, Facultad de Odontología, Universidad de Buenos Aires, Argentina. $\square$ (iD)

${ }^{2}$ Odontóloga, Especialista en Odontopediatría. Jefe de trabajos prácticos, Facultad de Odontología, Universidad de Buenos Aires, Argentina. $\triangle$ (iD)

${ }^{3}$ Odontólogo, Especialista en Odontopediatría. Jefe de trabajos prácticos, Facultad de Odontología Universidad de Buenos Aires, Argentina. $\bowtie$ (iD)

${ }^{4}$ Odontóloga, Especialista en Odontopediatría. Jefe de trabajos prácticos,

Facultad de Odontología, Universidad de Buenos Aires, Argentina. $\triangle$ (iD)

${ }^{5}$ Odontóloga, Especialista en Odontopediatría. Ayudante de primera, Facultad de Odontología, Universidad de Buenos Aires, Argentina. $\square$ (iD

${ }^{6}$ Licenciado en Ciencias Biológicas. Jefe de trabajos prácticos, Facultad de Odontología, Universidad de Buenos Aires, Argentina. $\square$ (iD)

${ }^{7}$ Odontóloga. Profesora adjunta, Facultad de

Odontología, Universidad de

Buenos Aires, Argentina. $\square$ (iD

${ }^{8}$ Doctor en Odontología. Profesor titular, Facultad de Odontología, Universidad de Buenos Aires, Argentina. $\bowtie$ (iD
RESUMEN Diferentes organismos internacionales han planteado la necesidad de un análisis de las condiciones de cada población para la identificación de los grupos e individuos de riesgo como estrategia operacional en la búsqueda de una mayor equidad, eficacia y eficiencia del sistema de salud. El objetivo de este trabajo fue identificar diferenciales en los perfiles de caries dental en la población escolar del partido de Avellaneda (provincia de Buenos Aires, Argentina) y su asociación con variables de protección social. En 2014, se realizó un estudio analítico observacional de corte transversal sobre una muestra de 656 escolares de 12 años de 40 escuelas públicas de los ocho distritos del partido (universo $=3580$ individuos distribuidos en 70 escuelas). El estado dentario de los escolares de 12 años en las localidades evaluadas mostró una distribución desigual, se identificaron subconjuntos poblacionales con elevada prevalencia, morbilidad y severidad de caries, que requieren focalización de las intervenciones sanitarias. Se observó asociación entre enfermedad desatendida y carencia de protección social.

PALABRAS CLAVES Perfil de Salud; Caries Dental; Determinantes Sociales de la Salud; Política Social; Argentina.

ABSTRACT Different international organizations have indicated the need to analyze the conditions of each population in order to identify groups and individuals at risk as an operational strategy in pursuit of greater equity, efficacy and efficiency in the health system. The aim of this study was to identify differentials in the dental caries profiles of children attending public schools in the county of Avellaneda (Province of Buenos Aires, Argentina) and their association with variables of social protection. In 2014, an observational, analytical and cross-sectional study was performed with a sample of 656 students 12 years of age from 40 public schools in the 8 county districts (out of a total universe of 3580 individuals distributed in 70 schools). The dental status of 12-year-old schoolchildren living in the localities evaluated showed an unequal distribution; subgroups with high caries prevalence, morbidity and severity were identified, requiring the targeting of health interventions. An association between untreated disease and lack of social protection was observed.

KEY WORDS Health Profile; Dental Caries; Social Determinants of Health; Social Policy; Argentina. 


\section{INTRODUCCIÓN}

La salud bucal es un fuerte indicador socioeconómico y conductual que puede ser considerado al estudiar desigualdades en cuidados de salud ${ }^{(1)}$. El problema de mayor prevalencia en salud bucal es la caries dental, una enfermedad crónica evitable, acumulativa, considerada la principal causa de dolor y pérdida de piezas dentarias en sus estadíos mas avanzados, y cuyas secuelas tienen impacto en el largo plazo ${ }^{(2)}$.

El informe global de incidencia, prevalencia y años vividos con discapacidad debido a enfermedades (agudas y crónicas) y lesiones, realizado sobre 188 países, muestra que la comorbilidad ha aumentado de manera considerable y en términos absolutos en el período 1990-2013. El dolor dental debido a caries en dentición permanente se encontró entre las 12 enfermedades y lesiones que presentaron entre 100 millones y $1.000 \mathrm{mi}$ Ilones de casos incidentales en 2013. Entre las principales causas asociadas a enfermedades crónicas o lesiones con secuelas crónicas, la caries dental sin dolor en dentición permanente se encontró entre las 8 causas que afectan a más del $10 \%$ de la población mundial. Entre los problemas de salud que tienen bajo impacto global en términos de discapacidad pero alto impacto en términos de recursos y planificación sanitaria se encuentra el desdentamiento (de 1 al $10 \%$ de prevalencia). La caries en dentición primaria se encuentra entre las 17 enfermedades que incrementaron su incidencia en las últimas dos décadas ${ }^{(3)}$.

Distintos estudios epidemiológicos sugieren que la distribución de dicha enfermedad no es uniforme en las distintas poblaciones. Las diferencias sistemáticas y potencialmente evitables poblaciones o grupos poblacionales en uno o más aspectos de la salud, en términos sociales, económicos, demográficos o geográficos de la salud, se conocen como desigualdades en salud. La desigualdad es la manifestación mensurable de la inequidad, proceso intrínseco de la sociedad ${ }^{(4)}$, a menudo expresada por las diferencias en el riesgo de enfermar y morir. Almeida Filho(5) define la desigualdad como una diferencia con dimensionalidad, en la esfera colectiva o poblacional. En cuanto evidencia empírica de inequidad puede ser expresada a través de indicadores. Según Lopez et al., estos riesgos se originan, a su vez, en las condiciones heterogéneas de la existencia en el acceso a bienes y servicios ${ }^{(6)}$. Otros investigadores consideran que el concepto de desigualdad toma en cuenta las diferencias que tienen las personas en el acceso a los recursos, tanto en calidad como en cantidad ${ }^{(7)}$. El estudio de estos factores es importante porque el acceso a los recursos se relaciona con las condiciones de vida de las personas.

Las desigualdades sociales en las etapas de desarrollo infantil son uno de los principales factores contribuyentes a las desigualdades en la vida adulta y, en consecuencia, a la creación de un círculo de desventaja intergeneracional. Diferentes métodos han sido propuestos para medir desigualdades, los cuales requieren de un análisis crítico para su aplicación en cada contexto en particular.

En referencia a las enfermedades bucales, y en especial a la caries dental, se han detectado desigualdades sociales y con gradiente social de forma concordante, con todos los indicadores utilizados y en todas las edades ${ }^{(8)}$. Squassi et al. analizaron variables vinculadas a la pobreza y su relación con la salud bucodental de niños preescolares y escolares residentes en áreas urbanas y suburbanas de Buenos Aires. Allí hallaron un nivel de enfermedad dental significativamente mayor en los grupos con vulnerabilidad social, un mayor número de niños con alto riesgo y actividad cariogénica se registró en los vecindarios con mayor riesgo social ${ }^{(9)}$. Esta relación fue demostrada en países en desarrollo así como en grupos vulnerables de países desarrollados y con diferentes sistemas de salud ${ }^{(10,11,12,13,14,15,16)}$. La caries dental es una enfermedad trazadora tanto del estado bucal como de un fenómeno social como la pobreza $^{(17)}$.

Existe consenso en considerar a la pobreza como heterogénea, es decir, no es igual en todas las regiones del mundo y las 
personas que la sufren la viven de manera distinta, dependiendo del contexto sociocultural del cual forman parte. También es multidimensional, ya que involucra todas las dimensiones del ser humano: sociales, psicológicas, culturales, ideológicas, espirituales, intelectuales. Los grupos de hogares con privación se distinguen según el tipo y la intensidad de sus carencias, por lo que existen indicadores y métodos muy variados para medirla. Para el abordaje del componente social en este estudio, el equipo de trabajo se ha centrado en dos ejes conceptuales: la noción de pobreza urbana desde la perspectiva de heterogeneidad de Katzman ${ }^{(18)}$ y la concepción de cobertura/protección social como mecanismo para hacer frente a las desigualdades ${ }^{(19)}$. El enfoque de Katzman incorpora la estructura social como elemento explícito del marco conceptual con que se interpretan los fenómenos de pobreza. Así, la localización de los pobres dentro de esa estructura varía no solo según la profundidad de las brechas que los separan de otras categorías sociales en el mercado de trabajo, sino también según el grado de segmentación en cuanto a la calidad de los servicios de todo tipo y el grado de segregación residencial. Según este autor, estas consideraciones permiten ampliar el campo de comprensión de los fenómenos de pobreza más allá de los esquemas que la conciben como producto de las vicisitudes de la economía, o como resultado del portafolio de recursos de los hogares y de su capacidad de movilizarlos de manera eficiente.

Las disparidades de ingresos y las diferencias en cuanto a protecciones y estabilidad laboral también se manifiestan en la localización de las clases en el territorio urbano. De hecho, una de las expresiones más notorias de la reducción de los ámbitos de interacción informales entre distintos estratos socioeconómicos es la progresiva polarización en la composición social de los vecindarios. El resultado de estos procesos es un creciente aislamiento social de los pobres urbanos con respecto a las corrientes principales de la sociedad. Dicho aislamiento se convierte en un obstáculo importante para acumular los activos que se necesitan para dejar de ser pobre, lo que hace que la pobreza urbana socialmente aislada se constituya en el caso paradigmático de la exclusión social. En síntesis, la pobreza urbana reconoce un aislamiento social de los individuos producto de la segmentación en los planos laboral, educativo y residencial que se potencian mutuamente afectando el capital social ciudadano, individual y colectivo ${ }^{(18)}$.

Complementando esta perspectiva, se incluyó la dimensión de la cobertura/protección social, que representa una forma de incluir en las dimensiones el papel del Estado, es decir, la aportación que, de manera directa, el Estado brinda a su sociedad al posibilitar, mediante la cobertura social, el acceso a situaciones que permitan a las personas mejores condiciones ${ }^{(20)}$. Los sistemas de protección y cobertura social constituyen un mecanismo para hacer frente a las desigualdades. Tienen como objetivo permitir a las personas reaccionar frente a imprevistos de diversa índole y controlar ciertos niveles de riesgo o privación considerados inaceptables en la sociedad. Pretenden compensar la ausencia o la reducción considerable de los ingresos del trabajo, proporcionar asistencia a las familias con hijos, y proporcionar a las personas atención médica, vivienda, agua potable y saneamiento, educación o trabajo social. Puede tratarse de ayudas en efectivo o en especie, que van de pensiones a exoneraciones de gastos de usuario a servicios subvencionados. En la Argentina, el modelo de articulación entre empleo y protección social se vinculó a la idea de Estado de bienestar que surgió con fuerza a partir de los años cincuenta en distintos países europeos. En los fundamentos que dieron origen a los Estados de bienestar, las personas recorrían la ruta del empleo y, en ese recorrido, obtenían la protección que necesitaban frente a distintas situaciones, como la enfermedad o el retiro. La condición de beneficiario de una obra social constituye un indicador indirecto de empleo formal. El fuerte peso del empleo informal refuerza la ya existente inestabilidad laboral, la falta de institucionalidad, la falta de acceso a tecnología y a mercados ${ }^{(19)}$. En la 
Argentina, como en el resto de los países de América Latina los planes sociales proveen en su mayoría transferencias de ingresos no contributivas a grupos sociales pobres, que se caracterizan por presentar dificultades de inserción en el mercado laboral y que, por tanto, se constituyen en sectores vulnerables y/o excluidos de los sistemas de protección derivados de los empleos protegidos ${ }^{(21)}$.

Con relación a la salud bucal, las "nuevas metas para el año 2020" establecidas conjuntamente por la Federación Dental Internacional (FDI), la Organización Mundial de la Salud (OMS) y la International Association for Dental Research (IADR) en el año 2003 incluyen objetivos y focos de aplicación globales, con la intención de que cada gobierno o entidad, haga inicialmente un análisis de su situación y asigne recursos para establecer metas propias específicas y reales ${ }^{(22)}$. Alazraqui y Spinelli ${ }^{(23)}$ plantean la necesidad de generar estudios sobre desigualdades a nivel de microáreas (municipios, áreas programáticas, barrios) aptos para guiar la toma de decisiones y los procesos de trabajo a nivel local. Una de las limitaciones identificadas por Almeida Filho en los estudios sobre desigualdades en Latinoamérica es que muy pocos de los que se realizan a nivel local incluyen datos primarios ${ }^{(5)}$.

La carencia de información epidemiológica referida al estado dentario de la población que reside en el partido de Avellaneda, reconocida por el equipo que dio origen y llevó adelante esta investigación, constituye un primer paso para la exploración de las desigualdades en este espacio territorial. Estimar la magnitud del problema e identificar comportamientos diferenciales en el perfil dentario en grupos focalizados del partido de Avellaneda pretende contribuir a la toma de decisiones adecuada a las realidades del contexto. Por lo tanto, el objetivo de este estudio es identificar diferenciales en los perfiles de caries dental en la población escolar del partido de Avellaneda (provincia de Buenos Aires) y su asociación con variables de protección social.

\section{MATERIALES Y MÉTODOS}

Se realizó un estudio analítico observacional de corte transversal. El diseño del estudio se observa en la Figura 1.

Para la selección de la muestra, se consideraron como criterios de inclusión: 1) edad 12 años, y 2) concurrencia a escuelas de gestión estatal del partido de Avellaneda (primer cordón del conurbano bonaerense). La edad de 12 años ha sido establecida por la Organización Mundial de la Salud como la edad global de vigilancia de la caries en comparaciones internacionales y de vigilancia de tendencias de la enfermedad, razón por la cual se seleccionaron escolares de esta edad para este estudio ${ }^{(24)}$. El recorte del universo del estudio, focalizado en las escuelas de gestión estatal, implica una mirada intencionada a los estratos de menores ingresos concentrados en estas escuelas. Este fenómeno es lo que Katzman describe como segmentación educativa de pobres urbanos ${ }^{(17)}$.

El universo estuvo constituido por 3.580 individuos distribuidos en 70 escuelas de gestión estatal a los que se invitó a participar del estudio. Aceptaron participar en el estudio 40 escuelas entre las que se distribuyeron los consentimientos informados. Finalmente, se obtuvo una muestra de 656 individuos de los ocho distritos de la localidad (Tabla 1).

\section{Variables e indicadores}

Se consideró como variable dependiente el estado dentario, y los aspectos analizados fueron:

- Morbilidad dentaria: Se caracterizó con el índice CPOD (cariado, perdido y obturado) con sus componentes discriminados (se estableció la participación porcentual de cada componente en el indicador global), y el índice ceod (cariado, extraído y obturado) ${ }^{(24)}$.

- Prevalencia de caries: porcentaje de individuos afectados.

- Severidad de caries: Se caracterizó con el recuento de individuos libres de caries 


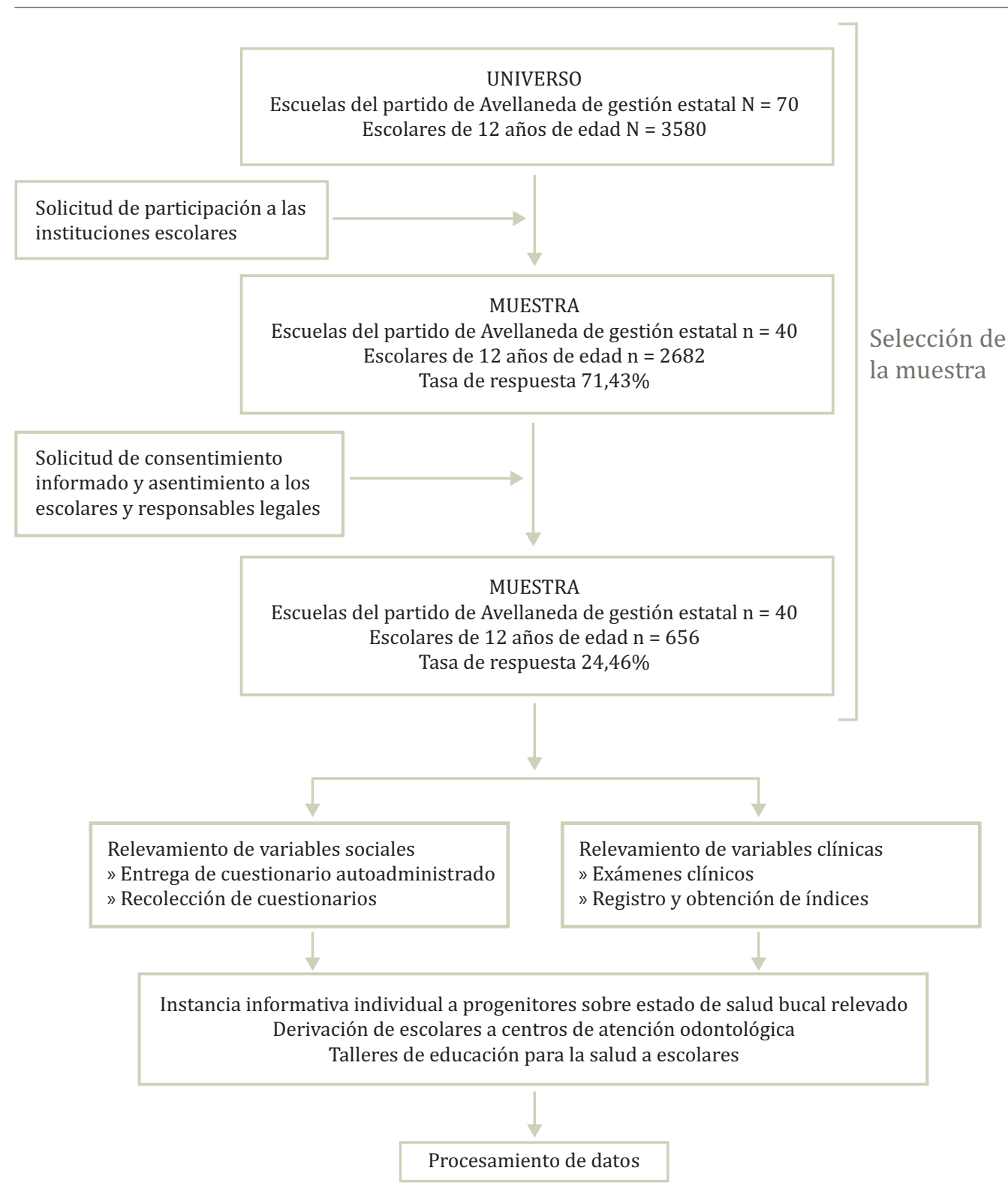

Figura 1. Proceso de conformación de la muestra y de la obtención de datos.

Fuente: Elaboración propia.

$(\mathrm{CPOD}+\operatorname{ceod}=0)$; con baja severidad de caries $(B S C)=$ CPOD $<3$ (el punto de corte para establecer baja severidad se estableció como el promedio del tercio de la población con menor valor de componente $C$ del CPOD, siendo 3 el puntaje); y con alta severidad de caries $=$ CPOD $>6$ (el punto de corte para establecer alta severidad se estableció como el promedio del tercio de la población con mayor valor de componente $C$ del CPOD, siendo 6 el puntaje).

Se consideraron como variables independientes la localidad (geográfica) de residencia; la condición de beneficiario de obra social; la condición de beneficiario de plan 
social del hogar. Esta selección se sustenta en que el estudio de la variable localidad o distrito propone dar cuenta del fenómeno de segmentación residencial, y que el estudio de la condición de beneficiario de obra social y planes sociales propone dar cuenta de una combinación de fenómenos como son la segmentación laboral junto con la cobertura/protección social como mecanismos del Estado que permiten hacer frente a las desigualdades ${ }^{(17,18,19)}$.

\section{Métodos para la recolección de datos}

Los diagnósticos clínicos para la determinación de los niveles de caries dental se realizaron en las instituciones educativas, en aulas con buena iluminación acondicionadas para tal fin.

Se utilizó el método visual-táctil para el diagnóstico de caries según los criterios propuestos por la OMS para desarrollo de estudios epidemiológicos ${ }^{(25)}$. A partir de los datos obtenidos se calcularon los indices CPOD y ceod con sus componentes discriminados individualmente según los criterios de Klein, Palmer, Knutson publicados por la $\mathrm{OMS}^{(26)}$, en 1997.

Cada escolar fue examinado en posición decúbito dorsal, utilizando espejo plano y explorador $\mathrm{N}^{\circ} 5$, con luz natural y linternas, $y$ secado de las superficies dentarias utilizando pipetas de goma y aislación relativa. Previo al examen clínico, con el fin de eliminar el biofilm dental (prerrequisito para el examen clínico) se realizó la enseñanza de la técnica de higiene bucal, en forma individual, seguida de un cepillado dental supervisado, con cepillos dentales nuevos proporcionados para tal fin.

Los examinadores fueron cuatro docentes de la Cátedra de Odontología Preventiva y Comunitaria de la Facultad de Odontología de la Universidad de Buenos Aires quienes, a su vez, formaban parte del personal de planta del Instituto Municipal de Odontología Infantil de Avellaneda (IMODI), calibrados con un investigador gold standard hasta alcanzar un Kappa $\geq 0,8$ interexaminador.
Tabla 1. Universo y muestra de niños de 12 años concurrentes a escuelas públicas del partido de Avellaneda, Provincia de Buenos Aires, 2014-2015.

\begin{tabular}{lrrrr} 
& \multicolumn{2}{c}{ Población } & \multicolumn{2}{c}{ Muestra } \\
\cline { 2 - 5 } Localidad & $\mathrm{n}$ & $\%$ & $\mathrm{n}$ & $\%$ \\
Dock Sud & 175 & 6,5 & 49 & 7,5 \\
Sarandí & 279 & 10,4 & 105 & 16,0 \\
Gerli & 280 & 10,4 & 81 & 12,3 \\
Wilde & 1.035 & 38,6 & 112 & 17,1 \\
Villa Domínico & 253 & 9,4 & 109 & 16,6 \\
Piñeyro & 161 & 6,0 & 126 & 19,2 \\
Avellaneda Centro & 499 & 18,6 & 74 & 11,3 \\
Total & & & & \\
& 2.682 & 100,0 & 656 & 100,0
\end{tabular}

Fuente: Elaboración propia.

Estos docentes contaron con la colaboración de estudiantes de $6^{\circ}$ año de la Facultad de Odontología de la Universidad de Buenos Aires, Cátedra de Odontología Preventiva y Comunitaria, entrenados y supervisados por sus docentes.

Los examinadores fueron asignados indistintamente a cada localidad. Los datos fueron recolectados en el período de marzo a noviembre de 2014 y de 2015. Los datos sobre cobertura/protección social se obtuvieron a partir de fuentes primarias mediante la aplicación de un cuestionario autoadministrado a las familias de los escolares.

\section{Cumplimiento de aspectos éticos}

Los tutores legales firmaron un consentimiento informado para autorizar la participación de de los escolares en este estudio. Se pidió el asentimiento formal de cada escolar para la realización de las acciones clínicas. La respuesta a los cuestionarios fue completamente voluntaria. El trabajo de investigación contó con la aprobación del comité de ética de la Universidad de Buenos Aires (UBACYT 20020120100324BA). 


\section{Procesamiento estadístico}

El estudio estadístico descriptivo incluyó, para las variables numéricas, el cálculo de la media aritmética y el desvío estándar, mediana y los percentiles 25 y 75 la población total y por localidades; $y$, para las variables dependientes categóricas, el cálculo de distribución de frecuencias y porcentajes.

El estudio estadístico bivariado y multivariado incluyó, para la comparación de variables cuantitativas ANOVA con pruebas robustas de Welch, aplicando pruebas post hoc de Games-Howell. Para la comparación de relación entre variables cualitativas se utilizó el test de independencia (Chi cuadrado). Para la comparación entre grupos se aplicó la prueba de proporciones independientes con aproximación a la normal o binomial según sea el caso aplicando corrección post hoc de Bonferroni. Para valorar el riesgo se aplicó el test de odds ratio y su intervalo de confianza al 95\%.

En todos los casos los test estadísticos aplicados fueron para muestras independientes.
Se utilizó un nivel de significación menor del $5 \%$ para rechazar la hipótesis nula.

\section{RESULTADOS}

La muestra estuvo constituida por 656 escolares de 12 años que concurrían a escuelas públicas de todas las localidades del partido de Avellaneda.

La distribución de los individuos examinados según la condición de cobertura/protección social del hogar y la localidad en que se encuentra ubicada la escuela a la que concurrían se puede observar en la Tabla 2. En la localidad de Dock Sud la proporción de hogares de los escolares que no contaban con el beneficio de una obra social ni un plan social fue significativamente mayor que en el resto de las localidades $(p<0,05)$. En las escuelas públicas de la localidad de Avellaneda Centro la proporción de hogares con obra social y sin plan social es significativamente mayor que en el resto de las localidades $(p<0,05)$ (Tabla 2$)$.

Tabla 2. Distribución de niños de 12 años concurrentes a escuelas públicas del partido de Avellaneda según beneficio social, por localidad. Provincia de Buenos Aires, 2014-2015.

\begin{tabular}{lcccccccc} 
& \multicolumn{3}{c}{ Sin obra social $(\mathrm{n}=341)$} & \multicolumn{3}{c}{ Con obra social (n=293) } \\
\cline { 2 - 8 } Localidad & \multicolumn{2}{c}{ Sin plan social } & Con plan social & Sin plan social & Con plan social \\
\cline { 2 - 9 } & $\mathrm{n}$ & $\%$ & $\mathrm{n}$ & $\%$ & $\mathrm{n}$ & $\%$ & $\mathrm{n}$ & $\%$ \\
Avellaneda Centro & 17 & 2,7 & 6 & 0,9 & 49 & $7,7^{*}$ & 1 & 0,2 \\
Dock Sud & 30 & $4,7^{*}$ & 5 & 0,8 & 6 & 0,9 & 0 & 0,0 \\
Gerli & 28 & 4,4 & 10 & 1,6 & 30 & 4,7 & 9 & 1,4 \\
Piñeyro & 51 & 8,0 & 24 & 3,8 & 46 & 7,3 & 5 & 0,8 \\
Villa Dominico & 37 & 5,8 & 17 & 2,7 & 45 & 7,1 & 7 & 1,1 \\
Sarandi & 32 & 5,0 & 19 & 3,0 & 41 & 6,5 & 8 & 1,3 \\
Wilde & 42 & 6,6 & 23 & 3,6 & 46 & 7,3 & 0 & 0,0 \\
Total & 237 & 37,4 & 104 & 16,4 & 263 & 41,5 & 30 & 4,7
\end{tabular}

Fuente: Elaboración propia. Nota: Los porcentajes fueron calculados sobre el total de la muestra. Sin datos = 22 casos ( $\mathrm{n}=634$ ). ${ }^{*} \mathrm{p}<0,05$ (Test de chi cuadrado y prueba de comparación de proporciones independientes aplicando corrección post hoc de Bonferroni). 
Tabla 3. Medidas de tendencia central de piezas dentales afectadas por caries según morbilidad en niños de 12 años concurrentes a escuelas públicas del partido de Avellaneda, provincia de Buenos Aires, 2014-2015.

$\begin{array}{lccccc}\text { Estado dentario } & \text { Media } & \text { Mediana } & \begin{array}{c}\text { Desviación } \\ \text { estándar }\end{array} & \begin{array}{c}\text { Percentil } \\ 25\end{array} & \begin{array}{c}\text { Percentil } \\ 75\end{array} \\ \text { CPOD } & 2,95 & 2 & 2,7 & 1 & 4 \\ \text { C / CPOD } & 2,15 & 1 & 2,6 & 0 & 3 \\ \text { P / CPOD } & 0,17 & 0 & 0,6 & 0 & 0 \\ \text { O / CPOD } & 1,17 & 0 & 2,6 & 0 & 1 \\ \text { ceod+CPOD } & 3,51 & 3 & 2,9 & 1 & 5 \\ \end{array}$

Fuente: Elaboración propia. Sin datos $=4$ casos $(n=652)$.

La media de piezas dentarias permanentes afectadas por caries a los 12 años en la muestra del total de Avellaneda $(n=652)$ mostró una media de 2,95 (mediana de 2), mientras que la totalidad de dientes afectados por caries en la misma muestra fue de 3,5 (mediana de 3 ) (Tabla 3). El 53,52\% [IC95\% $(49,95 ; 57,08)]$ de los dientes afectados mostró enfermedad no tratada (componente $\mathrm{C}$ del CPOD).

La prevalencia de caries en la muestra total fue de $86 \%$ correspondiendo el $43,6 \%$ a situaciones de baja severidad, $13,3 \%$ de los

Tabla 4. Prevalencia de caries y distribución según severidad de caries en niños de 12 años concurrentes a escuelas públicas del partido de Avellaneda, provincia de Buenos Aires, 2014-2015.

$\begin{array}{lrc}\text { Estado dentario } & \mathrm{n} & \% \\ \text { Sin caries } & 90 & 13,8 \\ \text { Prevalencia de caries } & 562 & 86,2 \\ & & \\ \text { Severidad baja } & 268 & 43,6 \\ \text { Severidad media } & 297 & 29,3 \\ \text { Severidad alta } & 87 & 13,3 \\ & & \end{array}$

Fuente: Elaboración propia. Sin datos $=4$ casos $(n=652)$. casos correspondieron a situaciones de alta severidad (Tabla 4).

Respecto de la distribución de enfermedad por distrito, se observaron diferencias significativas en términos de morbilidad (Tabla 5) como de severidad (Tabla 6). La localidad en la que los escolares mostraron los mayores niveles de caries tanto de morbilidad (media de dientes afectados $>6$ ) como de severidad ( $>25 \%$ ) fue Dock Sud, siendo la población que cuenta con los menores niveles de protección social. Por el otro lado, los escolares examinados en las escuelas públicas de las localidades de Gerli, Sarandi y Piñeyro mostraron los niveles más bajos de prevalencia (medias menores a 3) y de severidad de caries (menor al 10\%).

Se observó que la condición de alta severidad de caries estuvo presente en un $16,2 \%$ de los escolares cuyos hogares no cuentan con el beneficio de una obra social, en contraste con un 9,8\% entre los escolares cuyos hogares cuentan con ese beneficio. EI OR fue de $1,78[$ IC95\% $(1,11 ; 2,88)]$, es decir que los/as niños/as sin obra social tienen casi el doble de chances de tener alta severidad de caries con respecto a los que no cuentan con ella.

En la Figura 2 puede observarse la brecha entre lesiones de caries tratadas (componente obturado del (POD) y caries no tratadas 
Tabla 5. Medidas de tendencia central y dispersión, según estado dentario y localidad, en niños de 12 años concurrentes a escuelas públicas del partido de Avellaneda, provincia de Buenos Aires, 2014-2015.

\begin{tabular}{|c|c|c|c|c|c|c|}
\hline \multirow{2}{*}{ Localidad } & \multicolumn{5}{|c|}{ Estado dentario (CPOD+ceod) } & \multirow{2}{*}{ Valor de $p^{*}$} \\
\hline & Media & Mediana & $\begin{array}{c}\text { Desviación } \\
\text { estándar }\end{array}$ & Percentil 25 & Percentil 75 & \\
\hline Piñeyro & 2,6 & 2 & 2,4 & 0 & 4 & \\
\hline Sarandí & 2,9 & 2 & 2,4 & 1 & 4 & \\
\hline Gerli & 2,9 & 3 & 2,2 & 1 & 4 & \\
\hline Villa Domínico & 2,9 & 2 & 2,6 & 1 & 4 & 0,001 \\
\hline Avellaneda Centro & 4,0 & 4 & 2,7 & 2 & 5 & \\
\hline Wilde & 4,7 & 4 & 3,3 & 2 & 6 & \\
\hline Dock Sud & 6,1 & 5 & 3,7 & 4 & 9 & \\
\hline
\end{tabular}

Fuente: Elaboración propia. Sin datos = 4 casos $(\mathrm{n}=652)$.

Tabla 6. Distribución de la prevalencia y de la severidad de caries, según localidad, en niños de 12 años concurrentes a escuelas públicas del partido de Avellaneda, provincia de Buenos Aires, 2014-2015.

\begin{tabular}{|c|c|c|c|c|c|}
\hline \multirow{2}{*}{ Localidad } & \multicolumn{4}{|c|}{ Severidad de caries (\%) } & \multirow{2}{*}{$\begin{array}{l}\text { Prevalencia de } \\
\text { caries (\%) }\end{array}$} \\
\hline & Sin Caries & Severidad Baja & Severidad Media & Severidad alta & \\
\hline Gerli & 11,1 & 53,1 & 29,6 & 6,2 & 88,9 \\
\hline Piñeyro & 27,0 & 42,1 & 23,8 & 7,1 & 73,0 \\
\hline Sarandi & 17,1 & 47,6 & 25,7 & 9,5 & 82,9 \\
\hline Villa Dominico & 17,4 & 54,1 & 17,4 & 11,0 & 82,6 \\
\hline Avellaneda Centro & 4,1 & 43,2 & 40,5 & 12,2 & 95,9 \\
\hline Wilde & 6,3 & 33,9 & 35,7 & 24,1 & 93,8 \\
\hline Dock Sud & 0,0 & 20,0 & 46,7 & 33,3 & 100,0 \\
\hline Media & 13,8 & 43,6 & 29,3 & 13,3 & 86,2 \\
\hline
\end{tabular}

Fuente: Elaboración propia. Sin datos $=4$ casos $(n=652)$. 


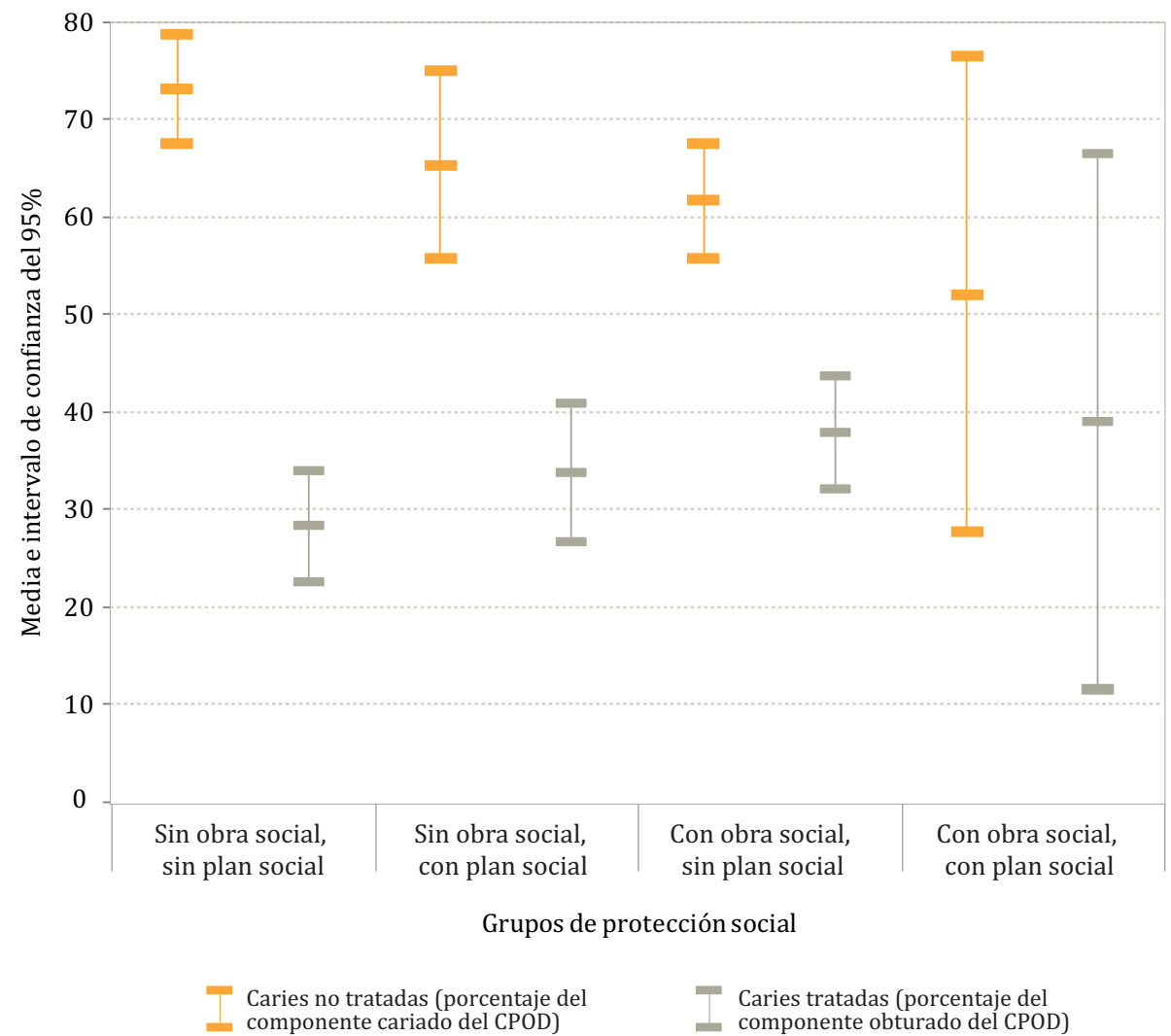

Figura 2. Participación porcentual de los componentes "cariado" y "obturado" en el indicador CPOD, en niños de 12 años concurrentes a escuelas públicas del partido de Avellaneda, provincia de Buenos Aires, 2014-2015.

Fuente: Elaboración propia.

(componente cariado del CPOD). En los sub-conjuntos poblacionales con menos beneficios de protección social se observó una brecha mayor entre piezas dentarias cariadas y obturadas.

\section{DISCUSIÓN}

Este estudio ha permitido caracterizar, por un lado, la magnitud de la morbilidad dentaria, prevalencia y severidad de caries entre escolares sobre el fin de la escolaridad primaria en el partido de Avellaneda (provincia de Buenos Aires) en el ámbito estatal. Por otro lado, se han identificado diferenciales en el perfil dentario en subconjuntos poblacionales correspondientes a un segmento de bajos ingresos según distritos y condición de cobertura/protección social.

Para establecer la magnitud de un problema de salud desde el punto de vista epidemiológico es necesario contar con un patrón de comparación. Ya en el año 1978, entre las metas para el año 2000, se estableció como deseable alcanzar una media de 3 o menos dientes cariados (CPOD) a la edad de 12 años $^{(27)}$. En el año 2006, el Comité Ejecutivo de la Organización Panamericana de la Salud planteó las siguientes categorías para establecer perfiles de caries en esa franja etaria: emergente (CPOD > 5 sin programas nacionales de fluoración de sal o agua); en crecimiento (CPOD 3-5 sin programas nacionales de fluoración de sal o agua); en 
consolidación (CPOD $<3$ con programas nacionales de fluoración de sal o agua) ${ }^{(28)}$. Desde esta perspectiva, los datos encontrados permiten encuadrar el perfil de caries de la muestra global estudiada del partido de Avellaneda en la categoría de menor nivel de patología ya que la media del CPOD encontrada fue menor a $3(2,95)$. Es de destacar, sin embargo, que alrededor de la mitad del componente corresponde a lesiones desatendidas $[53,52 \%$, IC95\% $(49,95 ; 57,08)]$, indicando una necesidad de atención no resuelta. Si se analiza el perfil epidemiológico discriminado por localidades se hacen visibles los diferenciales: de las localidades estudiadas, cuatro tienen perfiles de mayor nivel de patología, y tres en categoría "en crecimiento". En Dock Sud, se encontraron los mayores niveles de prevalencia, morbilidad y severidad de caries dental (categoría "emergente"). En coincidencia, esta localidad presenta los mayores niveles de hogares en situación de pobreza -según necesidades básicas insatisfechas (NBI) $(15,08 \%$, total partido $5,7 \%)-y$ con los menores niveles de protección social. El indicador NBI es un método directo para identificar carencias críticas en una población y caracterizar la pobreza que, en general, utiliza indicadores directamente relacionados con cuatro áreas de necesidades básicas de las personas (vivienda, servicios sanitarios, educación básica e ingreso mínimo), disponibles en los censos de población y vivienda. En la Argentina, se considera población con $\mathrm{NBI}$ a la que reúne alguna de las siguientes condiciones: más de tres personas viviendo en una misma habitación; alojamiento en viviendas precarias o de inquilinato; falta en la vivienda de retrete con descarga de agua; que en la familia exista algún niño entre 6 a 12 años que no vaya a la escuela.

Respecto de la proporción de escolares de 12 años libres de caries fue del 13,8\% con un rango de 0 a $27 \%$ según localidad. Piovano et al. ${ }^{(29)}$ reportaron en una población de igual edad, concurrentes a escuelas de la Ciudad Autónoma de Buenos Aires (Distritos Escolares 19, 21, 4 y 5) un valor para este indicador del 5,7\%, semejante a Avellaneda Centro y Wilde ( 4,1 y $6,3 \%$ respectivamente), situadas en distritos con alta densidad de hogares vulnerables ${ }^{(30)}$. El índice de vulnerabilidad social (IVS) es un índice ponderado que asigna a cada hogar un puntaje en función de su condición frente a un conjunto de características. Cada una de las siguientes dimensiones aporta un peso al puntaje total y puede subdividirse según la intensidad o profundidad del déficit: hacinamiento (moderado o crítico); material de la vivienda (déficit moderado o crítico); carga de dependencia por jubilado ( 2 o más personas en hogares sin ocupados); carga de dependencia por ocupado (5 o más personas en hogares sin jubilados); hogares que no reciben ingresos por trabajo ni jubilación ni pensión ${ }^{(31)}$.

En el contexto latinoamericano, los datos sobre distribución de caries dental muestran escasa sistematización. La Mesa de Representantes de Sociedades de Odontopediatría de América Latina en 2014 ofreció una síntesis de datos disponibles acerca de la situación bucodental de sus países. En nuestra investigación encontramos que la prevalencia de caries en escolares de 12 años $(86,2 \%$ con un rango que osciló entre 82,9 y $100 \%$ de individuos afectados) fue semejante a los datos reportados por los representantes de Perú, Paraguay, Venezuela y México ${ }^{(32)}$. En referencia a la morbilidad dentaria, Freire et al. ${ }^{(33)}$ reportaron para Brasil en 2010, en una cohorte de 12 años una media de CPOD de 2,04 con variaciones entre las ciudades capitales de distritos que oscilaron entre 0,77 en el sur (Florianópolis) y 4,15 en el norte (Porto Velho). En nuestro trabajo, los datos mostraron niveles de morbilidad mayores (media de 2,95 con un rango entre 2,6 y 6,1 ) que los de Brasil en 2010, y comparables con los de Paraguay en 2013 o Brasil en $2003^{(32)}$.

Los indicadores sociales utilizados para identificar desigualdades en los estudios epidemiológicos realizados hasta el momento han sido altamente variables. Los determinantes de la salud asociados a diferenciales en perfiles dentarios reportados en la bibliografía incluyen variables socioeconómicas como el nivel de escolaridad, ingresos familiares y ocupación de los miembros de la familia; variables contextuales como accesibilidad al 
sistema de salud y acceso a agua fluorada de consumo $^{(34,35,36)}$. En este trabajo hemos incluido la dimensión de cobertura/protección social como indicador indirecto de las condiciones laborales (acceso al trabajo formal asociado a la protección social bajo la forma de una obra social) y, por otro lado, de la protección social que provee el Estado como posible mecanismo para hacer frente a las desigualdades bajo la forma de planes sociales. En este sentido, este enfoque ha permitido observar una diferencia en la proporción de escolares con alta severidad de caries entre aquellos cuyos hogares cuentan con obra social (asociada al trabajo formal). La mayor proporción de lesiones dentarias desatendidas se concentró en el subconjunto que no contaba con ningún beneficio social del Estado. Un aspecto a considerar en este punto es que la muestra seleccionada para este estudio se focalizó intencionadamente en población concurrente a escuelas públicas, que concentran a los segmentos de población de menores ingresos. Trabajos realizados en Montevideo (Uruguay) $^{(36)}$ y Córdoba (Argentina) ${ }^{(37)}$ mostraron diferencias en el perfil dentario entre escolares de 12 años que concurren a escuelas públicas y privadas siendo mayores los niveles de afección en las escuelas de gestión estatal. Nuestros resultados evidencian heterogeneidades en los perfiles dentarios en este segmento poblacional.
Entre las limitaciones de este trabajo podemos hacer referencia al tipo de muestreo, el cual no fue representativo, sino por conveniencia. En este sentido, algunas localidades pueden encontrarse subrepresentadas o viceversa.

La importancia de este tipo de estudio radica en que, al identificar las diferentes variables referidas a la salud en un espacio geográfico poblacional pequeño, es posible identificar brechas sobre las que se puede intervenir de manera específica y diferenciada, así como monitorear los cambios que se van produciendo en los problemas identificados en cada territorio, según sus características particulares, que se reflejan a través de las modificaciones de los indicadores de salud. Si bien los datos relevados en este trabajo resultan insuficientes para establecer desigualdades, consideramos, sin embargo, que este estudio realizado con datos primarios, podría constituir un valioso insumo para la gestión en el nivel local.

En conclusión, este estudio ha permitido identificar subconjuntos poblacionales con elevada prevalencia, morbilidad y severidad de caries, que requieren focalización de las intervenciones sanitarias. Se observó asociación entre enfermedad desatendida y carencia de protección social.

\section{REFERENCIAS BIBLIOGRÁFICAS}

1. Thomson WM. Social inequality in oral health. Community Dentistry and Oral Epidemiology. 2012;40(Suppl 2):28-32. doi: 10.1111/j.16000528.2012.00716.

2. Oliveira LJC, Correa MB, Nascimento GG, Goettems ML, TarquínioSBC, Torriani DD, Demarco FF. Inequalities in oral health: are schoolchildren receiving the Bolsa Família more vulnerable? Revista de Saúde Pública. 2013;47(6):1-9.

3. Global Burden of Disease Study 2013 Collaborators. Global, regional, and national incidence, prevalence, and years lived with disability for 301 acute and chronic diseases and injuries in 188 countries, 1990-2013: a systematic analysis for the Global Burden of Disease Study 2013. The
Lancet. 2015;386(9995):743-800. doi: 10.1016/ S0140-6736(15)60692-4.

4. Breilh J. Epidemiología crítica: Ciencia emancipadora e interculturalidad. Buenos Aires: Lugar Editorial; 2003.

5. Almeida Filho N. Inequalities in health based on living conditions. Washington DC: OPS; 1999.

6. López E, Findling L, Abramzón M. Desigualdades en salud: ¿Es diferente la percepción de morbilidad de varones y mujeres? Salud Colectiva. 2006;2(1):61-74.

7. Borrell C, Benach J. Les desigualtats en la salut a Catalunya. Barcelona: Editorial Mediterrània; 2003. 
8. Font-Ribera L, García-Continente X, DavóBlanes MC, Ariza C, Díez E, García- Calvente MM, Maroto G, Suárez M, Rajmil L, Grupo de Determinantes Sociales de la Sociedad Española de Epidemiología. El estudio de las desigualdades sociales en la salud infantil y adolescente en España. Gaceta Sanitaria. 2014;28(4):316-325.

9. Squassi A, Mauro S, Mauro MJ, Sánchez G, Bordoni N. Relationship between oral health in children and poverty related factors. Acta Odontológica Latinoamericana. 2008;21(1):49-56.

10. Jones CM, Woods K, Taylor GO. Social deprivation and tooth decay in Scottish schoolchildren. Health Bulletin. 1997;55(1):11-15.

11. Vargas CM, Crall JJ, Schneider DA. Sociodemographic distribution of pediatric dental caries: NHANES III, 1988-1994. Journal of the American Dental Association. 1998;129(9):1229-1238.

12. Nainar SM. Longitudinal analysis of dental services provided to urban low-income (Medicaid) preschool children seeking initial dental care. ASDC Journal of Dentistry for Children. 1998;65(5):339-343, 355-356.

13. Sweeney PC, Nugent Z, Pitts NB. Deprivation and dental caries status of 5 -year-old children in Scotland. Community Dentistry and Oral Epidemiology. 1999;27(2):152-159.

14. Tickle $M$, Williams $M$, Jenner $T$, Blinkhorn $A$. The effects of socioeconomic status and dental attendance on dental caries' experience, and treatment patterns in 5-year-old children. British Dental Journal. 1999;186(3):135-137.

15. Kuriakose S, Joseph E. Caries prevalence and its relation to socio-economic status and oral hygiene practices in 600 pre-school children of KeralaIndia. Journal of the Indian Society of Pedodontics and Preventive Dentistry. 1999;17(3):97-100.

16. Prendergast MJ, Beal JF, Williams SA. The relationship between deprivation, ethnicity and dental health in 5-year-old children in Leeds, UK. Community Dental Health. 1997;14(1):18-21.

17. Bordoni N, Pistochini A, Squassi A. Epidemiología de caries dental en niños. En: Bordoni N, Castillo Mercado R. Odontología pediátrica: La salud bucal del niño y el adolescente en el mundo actual. Buenos Aires: Editorial Medica Panamericana; 2010. p. 881-892.

18. Katzman R. Seducidos y abandonados: el aislamiento social de los pobres urbanos. Revista de la CEPAL. 2001;75:171-189
19. Repetto F, Potenza dal Masseto F. Sistemas de protección social en América Latina y Caribe (LC/W.508). Buenos Aires: CEPAL; 2012.

20. Tovar JAO. Teorías del desarrollo y el papel del Estado: Desarrollo humano y bienestar, propuesta de un indicador complementario al índice de desarrollo humano en México. Política y Gobierno. 2014;XXI(2):409-441.

21. Gentile N. Funcionamiento de los planes sociales: ¿Dan respuesta a problemas de inserción laboral o fomentan la vagancia?, un estudio exploratorio a partir de indagar en las opiniones y expresiones de quienes reciben la asistencia social del Estado [Internet]. 2013 [citado 12 nov 2015]. Disponible en: http://tinyurl.com/gpuybw4.

22. Hobdell M, Petersen PE, Clarkson J, Johnson N. Global goals for oral health 2020. International Dental Journal. 2003;53:285-288.

23. Spinelli H, Alazraqui M. Abordaje conceptual de las desigualdades en salud en el nivel local/ municipal. En: Alazraqui M, Spinelli H. Desigualdades en salud en el nivel local/municipal. Lanús: Ediciones de la UNLa; 2008. p. 17-32.

24. World Health Organization. Continuous improvement of oral health in the $21 \mathrm{st}$. century the approach of the WHO Global Oral Health Programme. Geneva: WHO; 2003.

25. Klein H, Palmer CE, Knutson JW. Studies on dental caries: I dental status and dental needs of elementary school children. The Journal of the American Dental Association. 1938;25(10):1703-1705.

26. Organización Panamericana de la Salud. Salud oral (CD40/20) [Internet]. 1997 [citado 10 nov 2015]. Disponible en: http://tinyurl.com/ztbh83y.

27. Organización Mundial de la Salud. Atención Primaria de Salud: Informe de la Conferencia Internacional sobre Atención Primaria de Salud, Alma-Ata, URSS, 6-12 de septiembre de 1978. Ginebra: OMS; 1978.

28. Organización Panamericana de la Salud, Organización Mundial de la Salud. Propuesta de Plan Regional Decenal sobre salud bucodental (CE138/14) [Internet]. 2006 [citado 10 nov 2015]. Disponible en: http://tinyurl.com/za5csyd.

29. Piovano S, Bordoni N, Doño R, Argentieri A, Cohen A, Klemonskis G, Macucho Me, Pedemonte Z, Pistochini A, Squassi A. Estado dentario en niños, adolescentes y adultos de la Ciudad Autónoma de Buenos Aires. Revista de la Facultad de Odontología (UBA). 2008;23(54/55):34-42. 
30. Golovanevsky L. Vulnerabilidad Social: una propuesta para su medición en Argentina. Revista de Economía y Estadística. 2007;XLV(2):53-94.

31. Con M, Susini S, Catalá S, Quinteros S. Índice de vulnerabilidad social (IVS): Documento metodológico. En: Informes temáticos de la Dirección de Investigación y Estadística del Ministerio de Educación del GCBA [Internet]. 2011 [citado 10 nov 2015]. Disponible en: http://tinyurl.com/ jrp26x8.

32. Paiva SM, Vidigal EA, Abanto J, Cabrera-Matta A, López-Robles RA, Masoli C, Echevarría-Lopez SA, Mongelos-de-Idoyada MG, Guerra-Gamboa ME, Amado-Schneider AR. Epidemiología de la caries dental en América Latina. Revista de Odontopediatría Latinoamericana. 2014;4(2).

33. Freire $M C$, Reis SC, Figueiredo $N$, Peres KG, Moreira RS, Antunes JL. Individual and contextual determinants of dental caries in Brazilian 12-year-olds in 2010. Revista de Saúde Pública. 2013;47(Supl 3):40-49.
34. Organización Mundial de la Salud, Comisión sobre Determinantes Sociales de la Salud. Subsanar las desigualdades en una generación [Internet]. 2008 [citado 12 nov 2015]. Disponible en: http://tinyurl.com/j84jz8b.

35. Narvai PC, Frazão P, Roncalli AG, Antunes JLF. Cárie dentária no Brasil: declínio, polarização, iniqüidade e exclusão social. Revista Panamericana de Salud Pública. 2006;19(6):385-393. doi: 10.1590/S1020-49892006000600004.

36. Lorenzo S, Alvarez R. Prevalencia de caries en escolares de 12 años de diferente nivel socioeconómico, Montevideo, Uruguay, 2003. Odontoestomatología. 2009;11(13):27-36.

37. Yankilevich ERLM, Battellino L. Prevalencia de la caries dental en escolares de nivel primario de una región metropolitana de la Provincia de Córdoba, Argentina. Revista de Saúde Pública. 1992;26(6):405-413.

\section{FORMA DE CITAR}

Fort A, Fuks AJ, Napoli AV, Palomba S, Pazos X, Salgado P, Klemonskis G, Squassi A. Distribución de caries dental y asociación con variables de protección social en niños de 12 años del partido de Avellaneda, provincia de Buenos Aires. Salud Colectiva. 2017;13(1):91-104. doi: 10.18294/sc.2017.914.

Recibido: 30 de marzo de 2016 | Versión final: 23 de agosto de 2016 | Aprobado: 6 de octubre de 2016

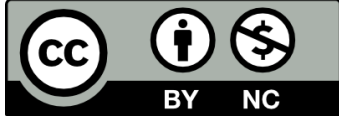

Este obra está bajo una licencia de Creative Commons Reconocimiento-NoComercial 4.0 Internacional. Reconocimiento - Permite copiar, distribuir y comunicar públicamente la obra. A cambio, se debe reconocer y citar al autor original. No Comercial - Esta obra no puede se utilizada con finalidades comerciales, a menos que se obtenga el permiso. 